Para enlazar con este artículo / To link to this article:

http://dx.doi.org/10.14198/fem.2019.34.09

Para citar este artículo / To cite this article:

Medina Arjona, Encarnación. «Ecofeminismo e interculturalidad». En Feminismo/s, 34 (diciembre 2019): 199-214. Dosier monográfico: Estado actual de la investigación en Literatura francesa y Género: balance y nuevas perspectivas / État présent de la recherche en Littérature française et Genre: bilan et nouvelles perspectives, coord. Ángeles Sirvent Ramos, DOI: 10.14198/fem.2019.34.09

\title{
ECOFEMINISMO E INTERCULTURALIDAD
}

ECOFEMINISM AND INTERCULTURALITY

\author{
Encarnación MEDINA ARJONA \\ Universidad de Jaén, Jaén \\ emedina@ujaen.es \\ https://orcid.org/0000-0002-3482-3748
}

\section{Resumen}

Proponemos una revisión del estado actual del ecofeminismo y de su íntima relación, señalada por Alicia Puleo, con la interculturalidad. Pondremos como ejemplo la Cátedra UNESCO de la UFGD (Brasil) donde se trabaja en estudios de género e interculturalidad.

Palabras clave: ecofeminismo; interculturalidad; naturaleza; patriarcado.

\begin{abstract}
We propose a review of the current state of ecofeminism and its intimate relationship, pointed out by Alicia Puleo, with interculturality. We will give as an example the UNESCO Chair of the UFGD (Brazil) where gender and intercultural studies are carried out.
\end{abstract}

Keywords: Ecofeminism; Interculturality; Nature; Patriarchy.

El término «ecofeminismo» fue creado por Françoise D’Eaubonne en 1974 en Le Féminisme ou la mort, pero se puede ver un germen del movimiento en 1962 con Rachel Carson en su Silent spring que, por su alcance, contribuyó a la prohibición del DDT en Estados Unidos. Se trata de una teoría y de un

Los contenidos de la revista se publican bajo una licencia de Creative Commons Reconocimiento 4.0 Internacional (CC BY 4.0)

Feminismo/s 34, diciembre 2019, pp. 199-214 
movimiento transnacional de origen anglosajón, que se constituyó para responder a la amenaza de la guerra nuclear entre la URSS y los EEUU. Agrupa, pues, militantes antinucleares, pero también ecologistas, feministas y pacifistas. Dio origen a movilizaciones espectaculares, como cuando en 1980 dos mil mujeres rodearon el Pentágono en la Women's Pentagon Action, o en 1981 el bloqueo de la central nuclear de Diablo Canyon, en California.

En Europa, el mayor campamento ecofeminista tuvo lugar en 1981, contra la instalación de misiles nucleares en Greeham Common, en Inglaterra. El corpus y las prácticas ecofeministas, que se despliegan desde hace cuarenta años en Estados Unidos, Australia, la India, y más recientemente en España, en América Latina, en Alemania y en Inglaterra, proporcionan importantes marcos teóricos y militantes para una renovación del feminismo. La variedad de tendencias dentro del ecofeminismo no impiden unos elementos comunes; un análisis crítico radical sobre el patriarcado, el capitalismo y el contexto materialista y técnico-científico de la mercantilización del ser vivo, así como la revolución verde de la agricultura industrial. En 1990, con el fin de la guerra fría, las movilizaciones se desinflan, pero en su artículo «The Power and the Promise of Ecological Feminism», Karen J. Warren puso de manifiesto las aportaciones y el enriquecimiento del ecofeminismo y la importancia del feminismo en la ética del medioambiente. En el COP23 (Conferencia de las Partes de la Convención Marco de Naciones Unidas sobre el Cambio Climático) en 2017, se adoptó el plan de acción «Género y clima»; en el COP24, en 2018, en Polonia, una empresa dirigida por mujeres en Haiti fue premiada por la ONU, por su proyecto que hablaba de «electrificación feminista». En 2019 el ecofeminismo está cada vez más presente en la sociedad. En plena movilización por el clima, en las marchas de Greta Thunberg, numerosas chicas han puesto toques feministas en sus pancartas volviendo a unir ecología y feminismo.

Desde el COP21 que tuvo lugar en París a finales de 2015, el movimiento ecofeminista suscita un repunte de interés en Europa. Jeanne Burgart Goutal, en «L'écoféminisme et la France: une inquiétante étrangeté?», repasa las últimas manifestaciones; cabe citar los Cahiers du genre, las revistas Terra Eco y Multitudes que han dedicado a ello un número recientemente; la colección «Sorcières», fundada por Isabelle Cambourakis, ha publicado algunos clásicos y una antología del ecofeminismo; los jóvenes colectivos militantes «Pieds de biche» y «EfFrontéEs» reclaman su pertenencia; la Cité des sciences et

Feminismo/s 34, diciembre 2019, pp. 199-214 
de l'industrie le dedicó en 2017 un cine-debate con el título «Écofeminisme, quand les femmes défendent la planète», en colaboración con el centro Hubertine Auclert para la igualdad, y la participación de Pascale d'Erm, autora de Sours en Écologie. La universidad comienza a interesarse, pero algunos estudios universitarios despolitizan el movimiento señalando únicamente la ética medioambiental. Émilie Hache acaba de dirigir la publicación de un libro, Reclaim, recueil de textes écoféministes, que recoge varios textos, todos ellos manifestando que el ecofeminismo tiene como objetivo la crítica y el cuestionamiento del capitalismo y el patriarcado, y en los que se muestra que todos sus combates ganadores han integrado preocupaciones medioambientales y de diversidad cultural, que la destrucción de la naturaleza y toda forma de opresión a las mujeres van íntimamente ligadas. Así, después de los trabajos de Carolyn Merchant, Silvia Federici señala recientemente en Caliban et la sorcière que el capitalismo, pasando del concepto de naturaleza sagrada a naturaleza que se puede dominar, percibe a las mujeres como seres inferiores que también pueden ser maltratadas. Ahora, las nociones de rehabilitación y de reinvención, traducidas por la noción de reclaim, responden al deseo de rechazar la naturaleza tal y como ha sido concebida por el patriarcado. Actualmente, hay diversidad de planteamientos ecofeministas, algunos relacionados con sentimientos religiosos o espiritualistas, otros más vinculados a la ecología social y a la filosofía. Como ejemplos, la corriente en torno a la figura de Vandava Shiva se articula sobre el tema socioeconómico, considerando que el patriarcado se encuentra íntimamente ligado a la dominación económica norte-sur; un ejemplo de inspiración de movimientos religiosos, en torno a Starhwak, en la que la mujer es parte integrante de la naturaleza y aspira a la no dominación del hombre sobre la mujer ni de los humanos sobre la naturaleza, es el pensamiento de la permacultura.

En España, destacamos los trabajos en ecofeminismo crítico de Alicia $\mathrm{H}$. Puleo. Claves ecofeministas. Para rebeldes que aman a la Tierra y a los animales da respuestas a la definición, origen y evolución del movimiento, y a cuestiones como la ética del cuidado, la educación ambiental o la violencia hacia los animales. Sus ensayos sobre ecofeminismo han sido publicados en España, Francia, Estados Unidos, Italia, Portugal, Brasil y otros países de América y Europa, y sus planteamientos han sido asumidos como base teórica por la Red Ecofeminista creada en Madrid en el año 2012. Como figura destacada en el

Feminismo/s 34, diciembre 2019, pp. 199-214 
ámbito hispánico, señalamos otros dos libros de la autora que consideramos básicos: Ecofeminismo para otro mundo posible y Ecología y género en diálogo interdisciplinar.

El extrañamiento frente a la naturaleza extrahumana, y a la vez nuestra confianza en/hacia ella, simultáneos, nos permiten, según Barbara HollandCunz (170), una aproximación a niveles cualitativos del conocimiento y de la simbolización. El concepto epistemológico de la «objetividad dinámica» desarrollado por Evelyn Fox Keller (121) es el que mejor se ajusta a la actitud y al modo de conocimiento que integra la autonomía y la ligazón, que garantiza la identidad de lo que se quiere llegar a conocer, al mismo tiempo que reconoce la ligazón que mantenemos con ello. La objetividad dinámica, según Keller (123), no es muy distinta de la empatía. Por su parte, Susan Griffin ha denominado esta forma de conocer «La actitud del respeto. La actitud del que todavía no sabe. La actitud del lento conocimiento» (228). Los planteamientos teórico-científicos feministas son los que señalan el camino que podría, o tendría, que llevar a un re-conocimiento de una lógica natural propia.

Janis Birkeland, en «Ecofeminism: Linking Theory and Practice», recuerda que el ecofeminismo es un sistema de valores, un movimiento social y una práctica, pero también ofrece un análisis político que explora los vínculos entre el androcentrismo y la destrucción ambiental. Es una «conciencia» (18) que comienza con la comprensión de que la explotación de la naturaleza está íntimamente vinculada a la actitud del hombre occidental hacia las culturas tribales y femeninas. En palabras de Ariel Salleh, sería que existe un «paralelo en el pensamiento de los hombres» entre sus «derechos» a explotar la naturaleza, por un lado, y el uso que hacen de las mujeres, por el otro (26).

En las culturas patriarcales dominantes, la realidad se divide según el género, y se asigna un mayor valor a los atributos asociados con la masculinidad, una construcción que se denomina «dualismo jerárquico» (Warren, «Feminism and Ecology» 17). En estas culturas, las mujeres han sido históricamente consideradas más cercanas al mundo, tierra o naturaleza. Además, las mujeres y la naturaleza han sido concebidas contra la mente y el espíritu, que en la cosmología occidental se han asociado con lo «masculino» y se han elevado a un plano superior del ser. Si bien solo podemos especular acerca de la conciencia patriarcal, es claro que se ha desarrollado una moralidad 
compleja basada en el dominio y la explotación en conjunción con la devaluación de la naturaleza y los valores «femeninos».

La asociación de las mujeres a la naturaleza es una construcción que aparece tanto en la cosmología de la Pachamama de Abya Yala como en cosmovisiones occidentales, así en la metafísica griega, que establece que el principio femenino está ligado a la materia/tierra por sus funciones de reproducción, y el del hombre a la técnica/cultura. De dicha identificación resulta que la mujer es más débil, exenta de derechos, excluida del poder y de la instrucción. La culminación llegó en el siglo XVII con la filosofía de Francis Bacon, invitando a los hombres a conquistar la tierra como si fuera el vientre de una mujer. Con un mismo movimiento, el Hombre domestica la naturaleza salvaje y, a la vez, a la mujer caprichosa y lunática, como señala Carolyn Merchant en The Death of Nature: Women, Ecology, and the Scientific Revolution.

Si bien el ecofeminismo proporciona un marco útil para el análisis político, quizás sea un proceso fundamentalmente activo. Para los y las ecofeministas, los valores y la acción son inseparables: el ser no puede preocuparse sin actuar. La teoría y el análisis ecofeministas solo se han desarrollado desde 1970, pero la práctica ha existido durante mucho más tiempo y ha crecido en muchas partes del mundo. Podemos citar el movimiento Chipko, de las campesinas del norte de la India que han luchado contra la tala de árboles; o de Lois Gibbs, la mujer que se movilizó contra los residuos tóxicos del Love Canal, en el estado de Nueva York, y que consiguió reglamentar los desechos químicos en la proximidad de las viviendas.

Desde las últimas décadas del siglo XX, algunas corrientes ecofeministas y ecologistas han dado relevancia a la figura de una mujer implicada con la Naturaleza. A partir de estudios antropológicos y multiculturales, y apoyados por los movimientos indígenas, se ha intentado luchar, con el abanderamiento de las mujeres, contra las complejas tramas tecnocientíficas modernas. Como señala Alicia Puleo en su importante libro Ecofeminismo para otro mundo posible, es necesario escuchar con atención el mensaje ecológico y feminista en América Latina. «Es el fruto inteligente del sincretismo y del diálogo entre la teoría y la praxis» (318). En dichos espacios latinoamericanos es imposible desligar el ecofeminismo de la interculturalidad, y así lo entiende también Alicia Puleo. Refiriéndose a la coexistencia de diversas culturas en un mismo espacio geográfico y un mismo tiempo histórico, el término

Feminismo/s 34, diciembre 2019, pp. 199-214 
«multiculturalidad» viene a designar el resultado de los flujos migratorios producidos por los desequilibrios económicos y ecológicos de las últimas décadas en numerosos países desarrollados. Pero también son realidades heredadas de antiguas colonizaciones, cuando los descendientes de los europeos llegados a tierras americanas a lo largo de los siglos constituyen sociedades multiculturales estratificadas según clasificaciones que combinan la clase social y la etnia. "Multiculturalidad es un concepto descriptivo, se limita a constatar un hecho. Multiculturalismo, en cambio, alude a una teoría con componentes normativos que nos dicen cómo han de relacionarse esas culturas.» (Ecofeminismo para otro mundo posible, 319). También Celia Amorós señala que el multiculturalismo, entendido como doctrina anti-ilustrada que exige respetar absolutamente todas las costumbres y evitar toda comparación crítica, es una interpretación abusiva de la Antropología, ya que cosifica las culturas, cuando en realidad las culturas nunca han estado aisladas, nunca han sido «cosas» inertes; siempre han ido evolucionando y todas tienen «conflictos y disensos» («Ética y Antropología» 184).

La gran diversidad cultural del mundo que rodea a las mujeres, marcada por la diversidad de lenguas, de formas de ver, de sentir, que hasta hace pocos años no hemos aprendido a apreciar en toda su magnitud, llevan a la categoría género a unirse indisolublemente a la perspectiva intercultural. Pero también las mujeres toman cada vez más conciencia de que viven en una realidad de grandes desigualdades sociales, económicas y culturales sobre las que es preciso reflexionar. Esas dos situaciones, diversidad y desigualdad, nos sitúan frente a nuevos retos. En los últimos tiempos, hemos oído hablar sobre la interculturalidad y los estudios de género. Hay un nuevo ambiente internacional compuesto por aspectos que ponen la interculturalidad y el género como un imperativo y una señal de los nuevos tiempos. Emergen una conciencia y una sensibilidad mayores para la diversidad cultural, como también un escenario que hace visibles las desigualdades de género, raza, etnia, etc., que emanan con el fenómeno de la globalización. Nuestro cuadro histórico actual presenta el proceso de «globalización» como representante de una política y estrategia civilizadora, impuesta como un proyecto para la humanidad, homogeneizadora, patriarcal y opositora a la propuesta intercultural. En ese contexto, concretamente en América Latina, la ausencia de un análisis a partir de una perspectiva de género no solo ocultaba las situaciones

Feminismo/s 34, diciembre 2019, pp. 199-214 
de discriminación que las mujeres viven en diferentes contextos culturales, sino que impedía dibujar un modelo educativo que educara para la igualdad.

Alicia Puleo señala que, a diferencia del multiculturalismo, el interculturalismo propone una apropiado contraste de las culturas que redunda en beneficio de todas ellas (Ecofeminismo para otro mundo posible 320). Sería lo que María Luisa Femenías llama «multiculturalismo crítico». La interpelación intercultural feminista desarrollada por Celia Amorós, «Feminismo y multiculturalismo», es una crítica que no tiene un único sentido. Dejando atrás el etnocentrismo, es una mirada que escucha, aprende y avanza gracias a la diversidad.

Tanto en Europa como en América Latina, la relación entre el feminismo y la interculturalidad es uno de los elementos esenciales para el desarrollo de una práctica intercultural crítica que pase a incorporar la igualdad de género como un fundamento ético inalienable. Así, hablar sobre interculturalidad y género tanto en comunidades indígenas como en comunidades campesinas en región de frontera es exponer muchos de los conflictos en nuestras sociedades, es aceptar que hay diferentes culturas y que los géneros no tienen el mismo reconocimiento y poder -señalamos aquí el libro de Aimé Tapia González, Mujeres indígenas en defensa de la tierra, que desgrana los rasgos de los diversos «feminismos indígenas» y la relación de los derechos de las mujeres a la tierra y al territorio. Fornet-Betancourt advierte que reconocer la diversidad requiere la conciencia de las desigualdades y de sus consecuencias: la pobreza, el sexismo, la discriminación, el racismo, la xenofobia, etc. Aquí encontramos otra razón poderosa para la interculturalidad: ayudar a cuestionar las situaciones estructurales y las condiciones que permiten que algunas culturas dominen sobre las otras, que unos grupos humanos sean preferidos sobre otros, que son, a su vez, rotulados como diferentes e inferiores. Es el caso, por ejemplo, de las comunidades indígenas y campesinas en la frontera con Paraguay y Bolivia que hace décadas sufren con la expropiación de sus tierras y son víctimas de constantes violencias de los propietarios de tierras.

Es necesario tener en cuenta, ya de antemano, la distancia entre lo que se dice y lo que se hace. Expresiones como la interculturalidad, al alojarse en el imaginario social como designativo de conductas políticamente correctas, pasan al nivel de la normatividad, o sea, pasan a ser parte de la moral, y pasan a construir realidades. Pero existe otro modo de ejercicio de

Feminismo/s 34, diciembre 2019, pp. 199-214 
la interculturalidad y el género; se trata de considerarlas como categorías analíticas, como un instrumento de análisis de los contactos establecidos entre los grupos humanos, pero también como proyecto político, buscando analizarlos a partir de sus presupuestos epistemológicos. Debatir las bases de una ética intercultural en un momento en que la globalización alcanza a prácticamente todas las gentes, desencadenando diversos tipos de procesos socioculturales en los ámbitos locales, que desembocan, algunas veces, en lo que Cullen llama «crisis morales», supone que hay un uso del concepto de interculturalidad que no cuestiona las reglas de la lógica del modelo económico neoliberal, llamado por Tubino «de interculturalidad funcional», que debe ser contrapuesto a la interculturalidad como proyecto ético-político de acción transformadora y democracia radical que sí denomina el de interculturalidad crítica. Las diferencias entre el interculturalismo funcional y el crítico, según el autor, no son nominativas, sino sustantivas. En Bolivia, el interculturalismo funcional es llamado interculturalismo neoliberal. En el mundo anglosajón, el interculturalismo no sería nada más allá del multiculturalismo de la acción afirmativa y discriminación positiva. Sin embargo, en líneas generales, el interculturalismo funcional busca promover el diálogo y la tolerancia sin hablar de las causas de la asimetría social y cultural vigente. Por otro lado, en el interculturalismo crítico, la búsqueda de supresión de esas asimetrías daría la tónica a la acción ético-política a través del uso de métodos políticos no violentos. El presupuesto defendido por el pensador es que el diálogo intercultural auténtico se haría inviable por la asimetría social y por la discriminación cultural y de género.

Así, antes del diálogo, habría que pensar en las condiciones sociales, económicas, políticas y culturales de ese diálogo, a fin de que no se caiga en la ideología de un diálogo descontextualizado, sexista y patriarcal, que favorecería solamente a los intereses creados por la racionalidad dominante. De ahí la necesidad del uso de un discurso de crítica social que dé visibilidad a las causas de la falta de diálogo. La interculturalidad crítica estaría, pues, constituida en los términos de una oferta ético-política de democracia inclusiva de la diversidad alternativa al carácter occidentalizante de la modernización social. La Interculturalidad como proyecto de democracia radical no es un antes sino un después de la Modernidad. Por ello, los filósofos que han reflexionado sobre el tema nos recuerdan que no hay que confundir la

Feminismo/s 34, diciembre 2019, pp. 199-214 
interculturalidad ni con el llamado nostálgico a un pasado idealizado que nunca existió (la utopía arcaica) ni con el rechazo maniqueo y en bloque a la Modernidad Occidental. Lo que se rechaza en la modernización es su sesgo homogeneizante y occidentalizador. Para Tubino, optar por la interculturalidad como proyecto de sociedad es optar por crear formas nuevas de modernidad. Lo que está en juego y en discusión, entonces, es la posibilidad de crear y recrear la modernidad desde múltiples tradiciones.

Así comprendemos que lo que desencadena la falta de respeto, la desigualdad, la violencia extrema a mujeres en regiones de América Latina está relacionado con una estructura de eventos que nos remiten a la propia historia del continente. La episteme eurocéntrica, unilateral, histórica y androcéntrica que hizo invisibles las otras formas de pensar y de relacionarse con el mundo, no aflora de una forma natural, y no cambiará si no es posible considerar otra racionalidad que se contraponga con las lógicas del mundo moderno, que finalmente comprenda y dialogue con sociedades diferentes, que envuelva epistemes diferentes, teniendo en cuenta las cuestiones de género.

En la historia de las sociedades humanas, las mujeres han sido percibidas y descritas no solo como diferentes, sino, sobre todo, como inferiores y ocupando un lugar inferior. Esa desigualdad se expresa en los sistemas simbólicos y de representación y se retroalimenta, reforzando un límite aparentemente insuperable y que separa actividades y conocimientos de hombres y mujeres.

A partir de las narrativas históricas tradicionales que desvaloraban el universo femenino, las propias mujeres alimentaron el equívoco de la equidad, aceptando como definición de un mundo igualitario aquel en que «solamente» tendrían que seguir siendo las mismas de siempre, añadiendo a sus vidas experiencias que, hasta entonces, eran propias del masculino. Entre los diversos movimientos sociales emergentes en los años 60, el movimiento feminista fue fundamental para un rompimiento de la racionalidad patriarcal y de las prácticas históricas de la discriminación contra la mujer. El movimiento feminista también ha sacado a la luz diversos problemas psicológicos, tales como la ansiedad, la soledad y los conflictos conyugales. Con ello, las mujeres se han dado cuenta de que tenían mucho en común y que podrían aprender de la Teoría Feminista.

Sobre Interculturalidad y Género se trabaja profundamente en la Universidade Federal da Grande Dourados (Brasil), desde su Cátedra 
UNESCO de Gênero, Diversidade Cultural e Fronteiras, con la que tenemos el placer de colaborar. El Movimiento Feminista en Brasil se desarrolló entre la clase media, y trajo propuestas de cambios profundos tanto en el sistema político como en las relaciones productivas y reproductivas de género, tales como la división sexual del trabajo, el aborto y la violencia sexual; además de preocuparse por los exiliados políticos. Surgida la Cátedra UNESCO desde el Departamento de Historia, fue entre las historiadoras que escribían sobre historia de las mujeres que la categoría de análisis «género» pasó a ser utilizada. Para ello, se inspiraron, en su mayoría, en el texto de Joan W. Scott, Gênero: uma categoria útil de análise histórica, publicado en Brasil en 1990.

Esa comprensión del género como una construcción se basa en aspectos sociales, culturales, económicos, políticos y psicológicos, ampliando las posibilidades de los enfoques históricos, trayendo a la discusión dos aspectos importantes: uno de ellos, la vinculación íntima del género con las relaciones de poder, y de otro, la definición de género como representación. Los dos no pueden ser admitidos o mensurados como elementos separados, estancos, divorciados, sino como constitutivos de realidades y acontecimientos históricamente situados. La percepción del género como una relación compleja y sociocultural implicaba que la acción de rastrear a las mujeres en la Historia no era simplemente una búsqueda de cierto aspecto antes olvidado; era más bien un problema de relaciones entre seres y grupos humanos que antes habían sido omitidas.

Para las pensadoras feministas, el uso de la categoría de análisis «género» significaba, como recordó en 1975 la historiadora Natalie Zemon Davis, combatir el discurso de la «naturaleza», lo que lleva a un determinismo biológico y se centra en la relación entre hombres y mujeres, que entendían los significados del género en el pasado. Las teorías de género pasan entonces a constatar que las relaciones entre culturas diferentes eran consideradas a partir de una lógica binaria (indio x blanco, centro x periferia, dominador $\mathrm{x}$ dominado, sur $\mathrm{x}$ norte, hombre $\mathrm{x}$ mujer, normal $\mathrm{x}$ anormal...), que no permitía comprender la complejidad de los agentes y de las relaciones implícitas en cada polo, ni la reciprocidad de las interrelaciones, ni la pluralidad y la variabilidad de los significados producidos en esas relaciones.

Los mismos puntos de vista críticos de las relaciones interculturales pueden ser tratados como entendimientos reductivos e inmovilizantes. De

Feminismo/s 34, diciembre 2019, pp. 199-214 
esa manera, si el concepto de dominación patriarcal y cultural se enreda en una lógica binaria y bipolar, puede llevar a suponer que los significados producidos por un sujeto social se determinan unidireccionalmente por la referencia cultural de otro sujeto. Esa comprensión puede fortalecer el proceso de sujeción a la medida que, al enfatizar la acción de un sujeto sobre otro, oscurece el hibridismo de las identidades, la ambivalencia y la reciprocidad de las relaciones sociales, así como la capacidad de autoría de diferentes sujetos sociales.

En el proceso de reconceptualización de la cultura y de reconocimiento de nuevas identidades culturales, hay una ruptura con la visión unilateral, homogénea, monolítica y patriarcal para construir una comprensión desde muchas miradas que interrumpen la linealidad y llegan a entender los procesos culturales como múltiples y discontinuos. De esa manera, el concepto de género niega la construcción universal de las diferencias sexuales e implica analizar la configuración de las relaciones sociales, envolviendo una perspectiva relacional, ya que las mujeres son concebidas a partir de sus relaciones con los hombres.

Si comprendemos la cultura como dinámica y transformadora, nos deberíamos preguntar: ¿por qué muchos de los conflictos culturales y violencias de género surgen por la percepción de que la conquista de la igualdad tiene como contrapartida la desintegración de los valores y de los referenciales tradicionales? Históricamente, la identidad cultural masculina fue fijada por el sometimiento de las mujeres. Alrededor de la dominación masculina y de la red de privilegios que concedidos históricamente, se elaboró una ideología para defender la supervivencia cultural recurriendo a la tradición como fuente principal de legitimación de la subordinación de las mujeres. La tradición siempre va al encuentro de esas prácticas, porque las mujeres han sido históricamente pactadas por los hombres, y bajo ese contrato sexual del que hablaba Carole Pateman se ha construido la división sexual del trabajo y de las funciones sexuales. Sin embargo, cuando la tradición no ayuda lo suficiente, ya que el poder de los hombres se debilita, algunos instrumentos culturales están en las manos de las mujeres y el pasado ha perdido legitimidad, entonces la cultura patriarcal inventa una nueva normatividad femenina que termina en la reactualización del viejo modelo de la mujer tradicional.

Feminismo/s 34, diciembre 2019, pp. 199-214 
Resaltamos que la naturalización de la dominación masculina, a partir de los esquemas de percepción, de los símbolos, de la cultura y de la oposición binaria entre el masculino y el femenino, dificulta el cuestionamiento de las desigualdades entre los sexos. La incorporación de la ideología patriarcal puede impedir que las mujeres tomen conciencia de su condición de sumisión y la enfrenten. La dominación masculina tiene todas las condiciones para su pleno ejercicio, una vez que las estructuras sociales, la división sexual del trabajo y de las funciones de producción y reproducción legitiman la supuesta superioridad masculina. La cultura, impregnada por la visión androcéntrica, da forma a las percepciones, a los pensamientos y a las acciones de todos los miembros de la sociedad. Siendo universalmente compartida, esa cultura asume un carácter trascendental e inmutable.

Evidentemente, tratando de cuestiones relativas a la superación de la desigualdad de género, ningún análisis podrá ser emprendido sin un intento de comprender cómo se construyen las relaciones de poder y las representaciones sociales, colocadas y jerarquizadas dentro de una sociedad o cultura en particular. El reconocimiento del plural, del diferente, del excéntrico, obligó la construcción de un nuevo concepto de cultura que hiciera referencia a lo que es complejo, múltiple, no lineal.

Hablar sobre relaciones de género bajo una perspectiva intercultural es comprender que la naturalización de las diferencias sexuales es la base de la exclusión de las mujeres de la ciudadanía y de la participación política.

Parece, pues, necesario deconstruir la disyuntiva creada entre igualdad y diferencia. De esa manera, se rechaza la selección entre igualdad o diferencia, denegando la idea de que constituyen una oposición, y el «dilema de la diferencia» se convierte un «falso dilema». Igualdad y diferencia comprendidas de ese modo no representan elecciones estratégicas a realizar por las feministas, porque estas no pueden renunciar ni a una cosa ni a la otra. Desde ese punto de vista, las diferencias deben ser tratadas no solo como externas, sino también como internas. Dicho de otra manera, no es suficiente discutir las diferencias entre hombres y mujeres (diferencias externas), es necesario también discutir las múltiples diferencias entre hombres y mujeres (diferencias internas). Si no nacemos hombres y mujeres, sino que somos construidos como tal, como ya ha dicho Simone de Beauvoir, esta construcción es histórica

Feminismo/s 34, diciembre 2019, pp. 199-214 
y social y, por lo tanto, hay múltiples maneras de construir lo que es ser un hombre y lo que es ser una mujer.

Así, la diferencia, la diversidad y las identidades en la historia de la humanidad tienden a ser esencializadas, cristalizadas y naturalizadas, pero ellas no tienen ese carácter esencial porque no son fijas, estables, únicas y permanentes, sino un efecto, una producción, una relación. La propuesta de una educación centrada en la diversidad sexual, étnica y racial nos pone a todos ante el gran desafío de estar atentos a las diferencias económicas, sociales y raciales, y de buscar el dominio de un conocimiento crítico que permita interpretarlas. La Cátedra UNESCO de la UFGD apuesta por que es necesario crear las condiciones que garanticen la igualdad de derechos en todas las esferas de la vida al mismo tiempo que el reconocimiento de la especificidad de cada cultura. Cuando se habla de sociedades indígenas de frontera, es necesario crear un ambiente que garantice la alteridad de cada localidad, teniendo como punto de partida la especificidad de cada grupo indígena y de cómo este crea/ inventa y transforma sus propias maneras de pensar sobre el mundo.

De ese modo, es lógico afirmar que cada cultura expresa su racionalidad a través de diversas representaciones que conducen al desarrollo de diferentes formas de percibir el mundo, las cuales pueden no parecer visiones «racionales» para los observadores externos. La escuela no puede alejarse de considerar que construye puentes entre las personas del mismo grupo social, y de ese grupo con otros grupos. Sin embargo, en el caso de los pueblos indígenas, es necesario darse cuenta de que esas relaciones no son exclusivas del contexto actual.

Pensar la interculturalidad desde la perspectiva crítica es uno de los caminos para luchar contra los prejuicios y las discriminaciones vinculadas al género, lo que constituye una nueva práctica cultural para una sociedad como la de América Latina, compuesta por diferentes grupos étnicos, en los que los rasgos de identidad, como el color de piel, las formas de hablar y la diversidad religiosa hacen la diferencia. Y esas marcas definen la movilidad, la condición social y los roles de género en nuestra sociedad.

Pensar las relaciones de género y la interculturalidad significa enfatizar la producción de los roles sexuales y sociales, significa exponerlos, hacerlos visibles, lo que lleva al cuestionamiento de los códigos, de las convenciones, de los artificios, de los discursos a través de los cuales las identidades de

Feminismo/s 34, diciembre 2019, pp. 199-214 
género son producidas: implica hacer visibles sus arquitecturas. Pensar la interculturalidad bajo la perspectiva del género es comprender que las dos son construcciones lingüísticas y discursivas, cuya realidad es propia.

Eso permitiría una visualización de las cuestiones de género y de las relaciones entre las culturas, constituidas como un elemento provocador de debate en el espacio público. Desde docentes en el aula hasta hombres y mujeres dedicados a la investigación histórica, se ha observado que es posible encontrar los caminos para visibilizar esas cuestiones. Hemos notado una preocupación por la igualdad, pero el hecho de reconocer los datos olvidados no supone que la experiencia histórica de las mujeres en su diversidad étnica, cultural, social y de clase sea necesariamente igual a la de los hombres. No se cuestiona ni siquiera la posibilidad de una experiencia diferente. ¿Cómo involucrar a los/las estudiantes activamente en ese proceso de organización y creación de un conocimiento histórico que subvierta las relaciones patriarcales de poder existentes y produzca una perspectiva intercultural no sexista? Es esencial una perspectiva que subvierta, contradiga, desestabilice los valores masculinos inscritos en la historia y en la comprensión de las formas por las cuales los mecanismos de producción de nuestras identidades sexuales se dirigen principalmente al cuerpo y al físico. Nuestros análisis de la enseñanza y el aprendizaje en espacios multiculturales han sido excesivamente racionalistas e intelectualistas. Pero si hay algo importante que nos fue enseñado por los estudios de género y por el movimiento feminista es que la inscripción de lo social en la producción de la subjetividad está inextricablemente ligada al sometimiento de nuestros cuerpos.

Solo en el contexto de una ética intercultural y de género será posible realizar el análisis de los conflictos inter e intra culturales. La Cátedra Unesco de la UFGD entiende que la historia de las mujeres contribuye a la narración y la revelación de una historia del silencio, una historia del confinamiento, más que del olvido. No basta, para hacer justicia al pasado, hacer una lista de las mujeres que constituyeron esa historia como si pudiéramos, por un artificio de archivo, de un acto de escribir dar sentido a la memoria y rescatar o enterrar simbólicamente a nuestras mujeres muertas, injustificadas, olvidadas. El futuro académico de la producción propia femenina, de las minorías sociales, depende de acciones de reanudación, rescate, salvación, del presente. La acción reflexiva, basada en la teoría crítica, necesita alcanzar a todas las

Feminismo/s 34, diciembre 2019, pp. 199-214 
personas promoviendo otra manera de hacer e interpretar la historia. En este sentido, la categoría género articulada con las demás herramientas de análisis de la historia social, es un instrumento valioso de re-descubrimientos epistemológicos, principalmente en espacios de frontera, de diversidad étnica, donde la interacción de varias culturas históricamente ha producido nuevas formas de leer y entender el mundo.

\section{REFERENCIAS BIBLIOGRÁFICAS}

Amorós, Celia. «Feminismo y multiculturalismo». Teoría feminista. De la Ilustración a la globalización, vol. 3. Eds. Celia Amorós y Ana de Miguel. Madrid: Minerva Ediciones, 2005. 215-264.

Amorós, Celia. «Ética y Antropología». La aventura de la moralidad. Eds. Carlos Gómez y Javier Muguerza. Madrid: Alianza editorial, 2007. 184-223.

Birkeland, Janis. «Ecofeminism: Linking Theory and Practice». Ecofeminism. Women, animals, nature. Ed. Greta Gaard. Philadelphia: Temple University Press, 1993. 13-59.

Burgart Goutal, Jeanne. «L'écoféminisme et la France: une inquiétante étrangeté?. Cités 73.1 (2018): 67-80.

Carson, Rachel. Silent spring. Boston: Houghton Mifflin, 1962.

Cullen, Carlos. Fenomenología de la crisis moral. Buenos Aires: Castañeda, 1978. Eaubonne, Françoise d'. Le Féminisme ou la mort. Paris: Pierre Horay, 1974.

Erm, Pascale d'. Sours en Écologie. Nantes: La Mer salée, 2017.

Federici, Silvia. Caliban et la sorcière. Paris: Entremonde, 2014.

Femenías, María Luisa. Multiculturalismo, identidad y violencia. Rosario: Prohistoria, 2013.

Fornet-Betancourt, Raúl. Interculturalidad y globalización. San José - Costa Rica: DEI, 2000.

Genre et environnement. Nouvelles menaces, nouvelles analyses au Nord et au Sud. Cahiers du Genre 59 (2015).

Griffin, Susan. Frau und Natur. Das Brüllen in ihr. Frankfurt am Main: Suhrkamp, 1987.

Hache, Émilie. Reclaim, recueil de textes écoféministes. Paris: Éditions Cambourakis, 2016.

Holland-Cunz, Barbara. Ecofeminismos. Madrid: Ediciones Cátedra / Universidad de Valencia, «Feminismos», 1996. 
Keller, Evelyn Fox. Liebe, Macht und Erkenntnis. Männliche oder weibliche Wissenschaft?. Múnich/Viena: Hanser, 1986.

Merchant, Carolyn. The Death of Nature: Women, Ecology, and the Scientific Revolution. New York: Paperback, 1980.

Multitudes 67 (2017). http://www.multitudes.net/category/l-edition-papier-enligne/67-multitudes-67-ete-2017/

Pateman, Carole. O contrato Sexual. São Paulo: Ed. Paz e Terra, 2005.

Puleo, Alicia H. Ecofeminismo para otro mundo posible. Madrid: Cátedra, 2011.

Puleo, Alicia H. Ecología y género en diálogo interdisciplinar. Madrid: Plaza y Valdés, 2015.

Puleo, Alicia H. Claves ecofeministas. Para rebeldes que aman a la Tierra y a los animales. Madrid: Plaza y Valdés, 2019.

Salleh, Ariel. «Stirrings of a New Renaissance». Island Magazine 8 (1989): 26-31.

Scott, Joan W. Gender: a useful category of historical analyses. Gender and the politics of history. New York: Columbia University Press, 1989.

Tapia González, Aimé. Mujeres indígenas en defensa de la tierra. Madrid: Cátedra, 2018.

Tubino, Fidel. «La interculturalidad crítica como proyecto ético-político». Encuentro continental de educadores agustinos, Lima, 24-28 de Enero 2005. https://oala.villanova.edu/congresos/educacion/lima-ponen-02.html

Warren, Karen J. «Feminism and Ecology: Making Connections». Environmental Ethics 9 (1987): 17-18.

Warren, Karen J. «The Power and the Promise of Ecological Feminism», Environmental Ethics 12 (1990): 121-146.

Zemon Davis, Natalie. «Women's History in Transition: the European Case». Feminist Studies 3.3 (1975) : 83-103.

«2016, l'année des écoféministes», Terra Eco 74 (2016). https://www.terraeco. net/2016-l-annee-des-ecofeministes,63260.html 